\title{
COMMENT
}

\section{Kappa counterconditioning of cocaine cues}

\author{
John R. Mantsch ${ }^{1}$ and Robert C. Twining ${ }^{1}$ \\ Neuropsychopharmacology (2018) 43:1469-1470; https://doi.org/10.1038/s41386-018-0037-5
}

Despite ongoing research efforts, drug addiction continues to pose a largely unmet medical need and is fueled by high rates of recidivism. The need for more effective interventions aimed at relapse prevention has led many researchers to re-evaluate current strategies and explore new and revisit old approaches to the problem. In their paper in the current issue of "Neuropsychopharmacology", Heinsbroek, Furbish and Peters use a rat model to explore the potential utility of an aversive "counterconditioning" approach, in which aversive stimuli are paired with drug associated cues/context with the goal of dampening their ability to elicit drug-seeking behavior. Using aversive conditioning to modify behavior is among the earliest ideas of psychology, dating back to the early 20th century when John B. Watson and Rosalie Rayner demonstrated that such an approach could be used to establish fear reactions to a previously rewarding stimulus (a lab rat) in a 9-month-old child.

In their paper, Heinsbroek and colleagues test the ability of the kappa opioid receptor (KOR) agonist, U50,488 (U50), a compound with established aversive properties, to counter-condition cocaine-associated cues, thereby attenuating their ability to evoke cocaine-seeking behavior. In the study, rats underwent repeated cocaine self-administration, followed by a 7-day abstinence period, after which they received a single dose of the KOR agonist or vehicle prior to re-introduction into the drug environment under extinction conditions in which responding no longer resulted in cocaine delivery. In addition to reducing responding during the initial retrieval session, the single U50 treatment significantly attenuated later reinstatement of cocaine seeking upon response-contingent cue presentation. This effect was dependent on KOR-induced activation of p38 MAP kinase signaling. The authors interpret their findings as reflecting KORmediated counterconditioning such that the new association between the aversive effects of the KOR agonist and the unrewarded operant response within the drug context counteracts the previously established cocaine association. They conclude that, to the extent that drug-associated stimuli can be isolated and introduced into a laboratory setting, KOR agonist delivery may be used to establish new aversive associations that reduce the likelihood of future drug-seeking behavior and relapse. Overall, the authors' findings are consistent with previous work demonstrating that KOR agonists can counteract the rewarding effects of cocaine itself (see e.g., refs. [1] and [2]).

The concept of using aversive conditioning to curb drug use is not a new one. Indeed, studies dating back to the 1970's have attempted to establish associations between aversive and drugrelated stimuli during therapy with the objective of reducing recidivism with limited success (see e.g., ref. [3]). However, several interesting observations by Heinsbroek and colleagues may better define how aversive stimuli or, more specifically, signaling processes engaged by aversive stimuli, may be better leveraged to reduce relapse risk.

The first observation is that the ability of KOR agonist administration prior to the retrieval session to "counter-condition" cue-induced cocaine seeking was not reproduced by lithium chloride ( $\mathrm{LiCl}$ ) administration at a dose that produces place aversion, reward devaluation, and gastric malaise. This finding was somewhat surprising, considering that KORs have been reported to mediate the aversive effects of stressors (see e.g., ref. [4]), although KOR mediation of $\mathrm{LiCl}$ effects has not been demonstrated. However, the finding is consistent with a previous report that the ability of footshock to "counter-condition" drug-cue associations resulting from cocaine self-administration is transient at best (see e.g., [5]). It may be that parallel processes engaged by $\mathrm{LiCl}$ and other stressors can offset or interfere with the effects of KOR activation that attenuate later drug seeking. Thus, the authors' findings suggest that specifically targeting the KOR and its downstream signaling mechanisms (e.g., p38/MAPK) can provide a pharmacological means for isolating components of the aversive response that can counteract or disrupt drug associations and attenuate later relapse susceptibility.

The second observation may provide insight regarding the precise KOR-regulated component of the aversive response that underlies the observed effect on cocaine seeking. The authors found that the ability of U50 to reduce later drug seeking was dependent not only on administration of U50 but also access to the previously active lever in the context suggesting a new or modified association is occurring that influences subsequent behavior. KORs are broadly distributed in areas of the brain that regulate associative learning, including the NAC and VTA. In fact, VTA KOR activation is required for both kappa agonist-induced reductions in NAC dopamine concentration and aversion learning [6]. The ability of KOR-induced signaling to inhibit dopamine neurons in the VTA [7] or alter downstream dopamine signaling via effects on NAc MSNs [8] could be particularly relevant to the authors' observations. It has long been proposed that a key function of dopamine is to encode error in reward prediction [9]. Elevated dopaminergic signaling in response to drug-predictive cues incentivize behavior while pauses in dopamine release are associated with reward omission and may reduce the likelihood of subsequent responding. In the latter case, an intriguing potential explanation for the observed response-dependent effects of U50 is that KOR activation produces a pharmacological enhancement of negative prediction error via suppressed dopaminergic signaling. Notably, suppression of dopamine signaling by KOR activation could also account for the acute attenuation of drug seeking by U50 during the initial retrieval session, as signaling in the nucleus accumbens has been implicated in the incentivizing properties of predictive cues. While appealing, this possible explanation has its

${ }^{1}$ Department of Biomedical Sciences, Marquette University, Milwaukee, USA
Correspondence: John R. Mantsch (john.mantsch@marquette.edu)

Received: 6 February 2018 Revised: 13 February 2018 Accepted: 15 February 2018

Published online: 6 March 2018 
limitations. First, $\mathrm{LiCl}$, which does not produce the same effects on later cue-induced cocaine seeking, has also been demonstrated to attenuate dopamine signaling [10]. However, the mechanism, duration, and intensity of $\mathrm{LiCl}$ action is vastly different than U50 and all of these factors are relevant for proper associative learning. Second, although p38 has been implicated in many KOR effects, it may not be required for the ability of KOR agonists to attenuate dopamine release, at least not stimulated dopamine release as measured using in vivo cyclic voltammetry in mice with a constitutive loss of p38 in midbrain dopamine neurons [7]. It is likely that both KOR-mediated reductions in dopamine signaling and KOR-mediated p38 activation are required for proper aversion learning. This raises the intriguing possibility that these distinguishable KOR-mediated signals may each contribute to different aspects of an animal's ability to learn about an aversive outcome. If this is the case, then discerning their respective contributions to this process could lead to the development of better therapeutics with fewer off-target dysphoric effects. Despite these limitations, the possibility that KOR regulation of dopaminergic signaling via actions in the VTA and/or nucleus accumbens contributes to the observed effects merits further investigation. Although the authors did not conduct brain region-specific manipulations in their paper, their findings establish an important starting point for a more targeted investigation of the precise mechanisms through which KOR agonists diminish the ability of drug-associated cues to elicit drug seeking.

Although the findings in the paper are important, there are a number of practical considerations that may limit immediate clinical application. First, ideal interventions involving counterconditioning approaches will require the identification and isolation of relevant drug-associated cues and their recapitulation in a therapy environment in a way that incorporates the operational act of drug seeking. While challenging, it is possible that such obstacles could be overcome through the use of guided imagery, possibly facilitated by modern technology such as virtual reality platforms. A bigger problem may be the limited efficacy of the approach. Extinction-based approaches that successfully mitigate drug-seeking behaviors often are limited to the extinction-context, thus latent or suppressed drug seeking behavior remains susceptible to renewal/relapse. Therefore, a demonstration that the kappa-mediated enhancement of operant extinction, when acquired outside of the drug context, carries over to reduced drug seeking within the original drug context would increase enthusiasm for kappa mediated counterconditioning approaches. Furthermore, although U50 administration significantly attenuated cue-induced drug seeking, blockade of reinstatement was incomplete. In human populations, where such levels of drug-seeking behavior will likely translate to use and, as a result, drug-cue associations will be re-established. Finally, the undesirable effects of KOR agonist drugs in human populations, which includes intense dysphoria and psychotomimetic effects has been well-documented and could limit clinical utility. However, as newer medications targeting KORs (e.g., biased agonists) and downstream signaling mechanisms are developed, the feasibility of using them therapeutically should increase.

In conclusion, while more work is needed to understand the underlying mechanism, the paper by Heinsbroek and colleagues raises the intriguing possibility that, to the extent that aversionbased approaches used in conjunction with extinction-based therapy represent a viable option for treating drug addiction, KOR agonist medications may provide an opportunity for more precise targeting of aversion-related processes that reduce the likelihood that drug-associated cues will serve as relapse triggers.

\section{ADDITIONAL INFORMATION}

Competing interests: The authors declare no competing interests.

Publisher's note: Springer Nature remains neutral with regard to jurisdictional claims in published maps and institutional affiliations.

\section{REFERENCES}

1. Schenk S, Partridge B, Shippenberg TS. U69593, a kappa-opioid agonist, decreases cocaine self-administration and decreases cocaine-produced drugseeking. Psychopharmacol (Berl). 1999;144:339-46.

2. Tomasiewicz HC, Todtenkopf MS, Chartoff EH, Cohen BM, Carlezon WA Jr. The kappa-opioid agonist U69,593 blocks cocaine-induced enhancement of brain stimulation reward. Biol Psychiatry. 2008;64:982-8.

3. Copemann CD. Drug addiction: II. An aversive counterconditioning technique for treatment. Psychol Rep. 1976;38:1271-81.

4. Bruchas MR, Land BB, Aita M, Xu M, Barot SK, Li S, Chavkin C. Stress-induced p38 mitogen-activated protein kinase activation mediates kappa-opioid-dependent dysphoria. J Neurosci. 2007;27:11614-23.

5. Tunstall BJ, Verendeev A, Kearns DN. A comparison of therapies for the treatment of drug sues in male rats: counterconditioning vs. extinction. Exp Clin Psychopharmacol. 2012;20:447-453.

6. Chefer VI, Bäckman CM, Gigante ED, Shippenberg TS. Kappa opioid receptors on dopaminergic neurons are necessary for kappa-mediated place aversion. Neuropsychopharmacology. 2013;38:2623-31.

7. Ehrich JM, Messinger DI, Knakal CR, Kuhar JR, Schattauer SS, Bruchas MR, Zweifel LS, Kieffer BL, Phillips PE, Chavkin C. Kappa opioid receptor-induced aversion requires p38 MAPK activation in VTA dopamine neurons. J Neurosci. 2015;35:12917-31.

8. Al-Hasani R, McCall JG, Shin G, Gomez AM, Schmitz GP, Bernardi JM, Pyo CO, Park SI, Marcinkiewcz CM, Crowley NA, Krashes MJ, Lowell BB, Kash TL, Rogers JA, Bruchas MR. Distinct subpopulations of nucleus accumbens dynorphin neurons drive aversion and reward. Neuron. 2015;87:1063-77.

9. Hollerman JR, Schultz W. Dopamine neurons report an error in the temporal prediction of reward during learning. Nat Neurosci. 1998;1:304-9.

10. Fortin SM, Chartoff EH, Roitman MF. The aversive agent lithium chloride suppresses phasic dopamine release through central GLP-1 receptors. Neuropsychopharmacology. 2016;41:906-15. 